\title{
Variants in the SMARCA4 gene was associated with coronary heart disease susceptibility in Chinese han population
}

\author{
Xuan Guo ${ }^{1,2, *}$, Xiaohong Wang ${ }^{2, *}$, Yuan Wang ${ }^{3}$, Chunyan Zhang ${ }^{1}$, Xiaohui Quan ${ }^{2}$, \\ Yan Zhang ${ }^{1}$, Shan Jia ${ }^{1}$, Weidong Ma ${ }^{1}$, Yajie Fan ${ }^{1}$ and Congxia Wang ${ }^{1}$ \\ ${ }^{1}$ Department of Cardiovascular Medicine, The Second Affiliated Hospital of Xi'an Jiaotong University, Xi'an, Shaanxi 710004, \\ China \\ 2 Department of Cardiovascular Medicine, The First Hospital of Xi'an, Xi'an, Shaanxi 710004, China \\ ${ }^{3}$ The Department of Orthopedics, People's Hospital of Manzhouli City, Manzhouli, Inner Mongolia 021400, China \\ * Joint first authors \\ Correspondence to: Congxia Wang, email:wcx622@163.com \\ Keywords: SMARCA4, coronary heart disease, gene polymorphisms, case-control study, Pathology Section \\ Received: August 04, $2016 \quad$ Accepted: November 21, $2016 \quad$ Published: December 30, 2016
}

\section{ABSTRACT}

Background: Coronary heart disease (CHD) is the leading cause of death worldwide. Many single-nucleotide polymorphisms (SNPs) are found to be related to the risk of CHD in previous studies. This study investigated whether polymorphism of SMARCA4 gene is associated with CHD.

Materials and methods: Genotypes at five CHD-relevant SNPs were determined in 456 cases of incident CHD and 685 unaffected controls in Chinese Han population using $x^{2}$ test, genetic model analysis and haplotype analysis. We also analysis the differences in continuous variables among the subjects with three genotypes of related genes were assessed using the ANOVA.

Results: We identified two susceptibility SNPs in the SMARCA4 gene that were potentially associated with a decreased risk of CHD. We identified rs11879293 (OR, $0.74 ; 95 \% \mathrm{CI}, 0.59-0.96 ; P=0.012)$ and rs12232780 (OR, 0.70; 95\% CI, 0.54$0.90 ; P=0.005$ ) were associated with a decreased risk of CHD risk under the logadditive model adjusted by gender and age. Meanwhile, we also found that significant differences in glucose concentrations with rs11879293 and rs1122608 different genotype. Serum LDL-C and HDL-C were seen among the 3 genotypes of rs12232780 exist differences.

Conclusion: This study provides an evidence for polymorphism of SMARCA4 gene associated with CHD development in Chinese Han population.

\section{INTRODUCTION}

Coronary heart disease (CHD) is a leading cause of death worldwide. In particular, CHD results in 700000 deaths in China and 502000 deaths in the United States annually $[1,2]$. CHD is a common complex disease that both genetic factors and lifestyle contribute to the demonstration of the disorder. A significant proportion of CHD is explained by risk scores based on age, gender and modifiable risk factors such as blood lipid profile [3]. Meanwhile, CHD is considered to be approximately 40$50 \%$ heritable and family history is a predictive factor of CHD [4]. Apart from monogenic disease, genetic information is not resulted in appreciable improvements in prediction over non-genetic risk factors. Pharmacologic preventive therapies are aimed at those at high risk > $20 \% 10$-year risk of CHD. Using risk scores, $15-20 \%$ of myocardial infarction (MI) patients would be considered from a substantial population attributable fraction of CHD [5]. According to previous reports, genome-wide association studies (GWAS) have successfully identified a lot of genetic mutations and chromosomal loci that are associated with CHD [6-9].

The SMARCA4 gene encodes an ATP-dependent helicase BRG1 and it belongs to SWI/SNF (switching defective/sucrose nonfermenting) complex [10]. According to previous researches, SMARCA4 is one of the most broadly mutated subunits, developing an 
Table 1 Basic characteristics of this study population

\begin{tabular}{|c|c|c|c|}
\hline Parameters & Cases & Controls & $p$ \\
\hline No. & 456 & 685 & \multirow{3}{*}{0.010} \\
\hline Males & $291(63.8 \%)$ & $385(56.2 \%)$ & \\
\hline Females & $165(36.2 \%)$ & $300(43.8 \%)$ & \\
\hline Mean age & $61.17 \pm 11.86$ & $48.59 \pm 9.56$ & $p<0.001$ \\
\hline ALT (U/L) & $31.15 \pm 2.11$ & & \\
\hline AST (U/L) & $36.41 \pm 2.93$ & & \\
\hline GGT (U/L) & $44.65 \pm 3.77$ & & \\
\hline $\mathrm{TP}(\mathrm{g} / \mathrm{L})$ & $66.39 \pm 0.3$ & & \\
\hline GLU (mmol/L) & $6.34 \pm 0.11$ & & \\
\hline $\mathrm{TG}(\mathrm{mmol} / \mathrm{L})$ & $1.8 \pm 0.07$ & & \\
\hline $\mathrm{TC}(\mathrm{mmol} / \mathrm{L})$ & $4.09 \pm 0.06$ & & \\
\hline HDL-C (mmol/L) & $1.13 \pm 0.01$ & & \\
\hline LDL-C (mmol/L) & $1.92 \pm 0.04$ & & \\
\hline APOA1 $(\mathrm{g} / \mathrm{L})$ & $1.26 \pm 0.01$ & & \\
\hline $\mathrm{APOB}(\mathrm{g} / \mathrm{L})$ & $1 \pm 0.02$ & & \\
\hline $\mathrm{Lp}(\mathrm{a})(\mathrm{mg} / \mathrm{L})$ & $239.2 \pm 12.14$ & & \\
\hline PLT (109/L) & $169.42 \pm 3.56$ & & \\
\hline РCT $(\%)$ & $1.14 \pm 0.16$ & & \\
\hline MPV (fl) & $13.08 \pm 0.35$ & & \\
\hline PDW (\%) & $14.23 \pm 0.15$ & & \\
\hline
\end{tabular}

ALT: alanine aminotransferase, AST: aspartate aminotransferase, GGT: gamma-glutamyl

transpeptidase, TP: total protein, GLU: glucose, TG: triglyceride, TC: total cholesterol, HDL:

high-density lipoprotein, LDL: low-density lipoprotein, apoA: apolipoprotein A, apoB:

apolipoprotein B, LP(a): lipoprotein, PLT: platelet, PCT: plateletcrit, MPV: Mean Platelet Volume PDW: platelet distribution width.

$p<0.05$, statistical significance

understanding of the mechanisms by which mutation of SMARCA4 drives cancer and of the vulnerabilities created carries major disease relevance [11]. Fujimaki et al. [12] performed a case-control study to reveal the relation between SMARCA4 genetic polymorphisms and coronary artery disease $(\mathrm{CAD})$ in a Japanese population. Jamaldini et al. [13] found intron 30 of SMARCA4 gene include rs 1122608 was associated with CAD in Iranian population. Previous studies also identified rs11879293, rs12232780, rs2072382 and rs1529729 variants effected on lipid parameters and dyslipidemia related disease [12, $14,15]$. However, the role of SMARCA4 gene mutation in the development of CHD remains poorly understood in Chinese Han population. The primary aim of this analysis was to determine whether exist the relationship between polymorphisms of SMARCA4 gene and CHD in the Chinese Han population.

\section{RESULTS}

\section{Base information}

We included a total of 456 CHD patient cohorts consisted of 291 males and 165 females with a mean age of $61.17 \pm 11.86$ years at the time of diagnosis. The 685 healthy controls included 385 males and 300 females with a mean age of $48.59 \pm 9.56$ years at the time of study enrollment. We found no differences between gender or age distribution between the case and control groups. The characteristics of these patient cohorts are summarized in Table 1. Five SNPs in the SMARCA4 gene were analyzed in this study. Chromosomal, position, band, HWE $p$ value, alleles A/B, MAF control and MAF case for all the SNPs are shown in Table 2. The rs2072382 were cut off at 5\% HWE $p$ level. 
Table 2: Basic SNPs in SMARCA4 gene summary of all study participants

\begin{tabular}{|l|c|c|c|c|c|c|c|c|c|}
\hline SNP ID & $\begin{array}{l}\text { Alleles } \\
\text { A/B }\end{array}$ & Chromosome & Position & Band & $\begin{array}{l}\text { HWE } \\
\text { value }\end{array}$ & $\begin{array}{l}\text { MAF } \\
\text { control }\end{array}$ & $\begin{array}{l}\text { MAF } \\
\text { case }\end{array}$ & OR(95\%CI) & $\boldsymbol{p}$ value \\
\hline rs11879293 & $\mathrm{A} / \mathrm{G}$ & 19 & 11072610 & $19 \mathrm{p} 13.2$ & 0.5577 & 0.266 & 0.240 & $0.87(0.72-1.06)$ & 0.17 \\
\hline rs12232780 & $\mathrm{A} / \mathrm{G}$ & 19 & 11132080 & $19 \mathrm{p} 13.2$ & 0.2722 & 0.224 & 0.194 & $0.83(0.68-1.03)$ & 0.09 \\
\hline rs2072382 & $\mathrm{T} / \mathrm{C}$ & 19 & 11152650 & $19 \mathrm{p} 13.2$ & $0.0100^{*}$ & 0.279 & 0.326 & $1.25(1.04-1.50)$ & 0.02 \\
\hline rs1529729 & $\mathrm{C} / \mathrm{T}$ & 19 & 11163562 & $19 \mathrm{p} 13.2$ & 0.1273 & 0.228 & 0.220 & $0.96(0.78-1.17)$ & 0.69 \\
\hline rs1122608 & $\mathrm{T} / \mathrm{G}$ & 19 & 11163601 & $19 \mathrm{p} 13.2$ & 0.256 & 0.093 & 0.084 & $0.90(0.67-1.21)$ & 0.50 \\
\hline
\end{tabular}

A/B stands for minor/major alleles on the control sample frequencies.

SNPs are excluded at 5\% HWE $P$ level

$p<0.05$ indicates statistical significance

$p<0.01$ indicates statistical significance for multiple comparison

Table 3: Association of SNPs with coronary heart disease risk based on logistical tests

\begin{tabular}{|c|c|c|c|c|c|c|c|c|}
\hline \multirow{2}{*}{ SNP } & \multirow{2}{*}{ Model } & \multirow{2}{*}{ Genotype } & \multirow{2}{*}{ control } & \multirow{2}{*}{ case } & \multicolumn{2}{|c|}{ crude analysis } & \multicolumn{2}{|c|}{ adjusted analysis } \\
\hline & & & & & OR $(95 \%$ CI $)$ & $P$-value & OR (95\% CI) & $P$-value \\
\hline \multirow{8}{*}{ rs11879293 } & Codominant & $\mathrm{G} / \mathrm{G}$ & $365(53.4 \%)$ & $262(57.5 \%)$ & 1 & 0.380 & 1 & 0.041 \\
\hline & & $\mathrm{G} / \mathrm{A}$ & $273(40 \%)$ & $169(37.1 \%)$ & $0.86(0.67-1.11)$ & & $0.73(0.54-0.99)$ & \\
\hline & & $\mathrm{A} / \mathrm{A}$ & $45(6.6 \%)$ & $25(5.5 \%)$ & $0.77(0.46-1.29)$ & & $0.57(0.31-1.04)$ & \\
\hline & Dominant & $\mathrm{G} / \mathrm{G}$ & $365(53.4 \%)$ & $262(57.5 \%)$ & 1 & 0.180 & 1 & 0.016 \\
\hline & & G/A-A/A & $318(46.6 \%)$ & $194(42.5 \%)$ & $0.85(0.67-1.08)$ & & $0.71(0.53-0.94)$ & \\
\hline & Recessive & G/G-G/A & $638(93.4 \%)$ & $431(94.5 \%)$ & 1 & 0.440 & 1 & 0.140 \\
\hline & & $\mathrm{A} / \mathrm{A}$ & $45(6.6 \%)$ & $25(5.5 \%)$ & $0.82(0.50-1.36)$ & & $0.65(0.36-1.17)$ & \\
\hline & Log-additive & --- & --- & --- & $0.87(0.72-1.06)$ & 0.160 & $0.74(0.59-0.94)$ & 0.012 \\
\hline \multirow{8}{*}{ rs 12232780} & Codominant & $\mathrm{G} / \mathrm{G}$ & $406(59.4 \%)$ & $292(64 \%)$ & 1 & 0.200 & 1 & 0.019 \\
\hline & & $\mathrm{G} / \mathrm{A}$ & $248(36.3 \%)$ & $151(33.1 \%)$ & $0.85(0.66-1.09)$ & & $0.72(0.53-0.97)$ & \\
\hline & & $\mathrm{A} / \mathrm{A}$ & $29(4.2 \%)$ & $13(2.8 \%)$ & $0.62(0.32-1.22)$ & & $0.43(0.19-0.98)$ & \\
\hline & Dominant & $\mathrm{G} / \mathrm{G}$ & $406(59.4 \%)$ & $292(64 \%)$ & 1 & 0.120 & 1 & 0.011 \\
\hline & & G/A-A/A & $277(40.6 \%)$ & $164(36 \%)$ & $0.82(0.64-1.05)$ & & $0.69(0.51-0.92)$ & \\
\hline & Recessive & G/G-G/A & $654(95.8 \%)$ & $443(97.2 \%)$ & 1 & 0.210 & 1 & 0.072 \\
\hline & & $\mathrm{A} / \mathrm{A}$ & $29(4.2 \%)$ & $13(2.8 \%)$ & $0.66(0.34-1.29)$ & & $0.49(0.22-1.09)$ & \\
\hline & Log-additive & --- & --- & -- & $0.83(0.67-1.02)$ & 0.078 & $0.70(0.54-0.90)$ & 0.005 \\
\hline
\end{tabular}

$p<0.05$ indicates statistical significance

$p<0.0063$ indicates statistical significance for multiple comparison

\section{SMARCA4 gene and CHD}

Using logistic test, four models (recessive, dominant, codominant and log-additive) were applied for analyzing the association of SNPs and CHD risk by listed in Table 3. We identified rs11879293 was associated with a decreased risk of CHD risk under the log-additive model (OR, 0.74; 95\% CI, 0.59-0.96; $P=0.012$ ) adjusted by gender and age. We also identified rs12232780 was associated with a decreased risk of CHD risk under the logadditive model (OR, 0.70; 95\% CI, 0.54-0.90; $P=0.005$ ) adjusted by gender and age. Used multiple comparison, eight tests were performed, a significance level was $P<$ $0.0063(0.05 / 8)$ based on Bonferroni correction, we found that rs 12232780 in SMARCA4 gene influence the risk of Coronary heart disease. 
Table 4: Clinical and biochemical characteristics of participants, stratified by SMARCA4 gene polymorphism status

\begin{tabular}{|l|c|c|c|c|}
\hline \multicolumn{1}{|c|}{ SNP } & \multicolumn{3}{c|}{ Genotype } & $\boldsymbol{p}$ \\
\hline \multicolumn{1}{|c|}{ rs11879293 } & GG & GA & AA & \\
\hline GLU & $6.18 \pm 2.21$ & $6.36 \pm 2017$ & $7.79 \pm 4.82$ & 0.006 \\
\hline TC & $3.99 \pm 1.04$ & $4.29 \pm 1.30$ & $3.96 \pm 1.28$ & 0.033 \\
\hline LDL-C & $1.80 \pm 0.65$ & $2.12 \pm 1.07$ & $1.84 \pm 0.69$ & 0.001 \\
\hline rs12232780 & GG & GA & AA & \\
\hline HDL-C & $1.13 \pm 0.25$ & $1.14 \pm 0.26$ & $0.95 \pm 0.14$ & 0.047 \\
\hline LDL-C & $1.84 \pm 0.69$ & $1.84 \pm 0.69$ & $1.67 \pm 0.58$ & 0.012 \\
\hline rs1529729 & TT & TC & CC & \\
\hline PDW & $14.38 \pm 3.03$ & $13.85 \pm 2.71$ & $15.63 \pm 3.08$ & 0.043 \\
\hline rs1122608 & GG & GT & TT & \\
\hline GLU & $6.20 \pm 2.131$ & $7.08 \pm 3.60$ & $6.65 \pm 1.45$ & 0.019 \\
\hline
\end{tabular}

$p<0.05$ indicates statistical significance

$p<0.0071$ indicates statistical significance for multiple comparison

\section{SMARCA4 gene and clinical information}

The basic characteristics of the study subjects stratified by different genotype are shown in Table 4 . We also found significant differences in glucose concentrations with SMARCA4 rs11879293 and SMARCA4 rs1122608 different genotype. For rs11879293, the mean ( \pm Std) serum glucose concentration was $6.18 \pm 2.21 \mathrm{mmol} / \mathrm{L}$ for the GG genotype (lowest), $6.36 \pm 2.17 \mathrm{mmol} / \mathrm{L}$ for the GA genotype, $7.79 \pm 4.82 \mathrm{mmol} / \mathrm{L}$ for the AA genotype (highest) $(p=0.006)$. For rs 1122608 , the mean $( \pm \mathrm{Std})$ serum glucose concentration of GG, GT and TT is $6.20 \pm$ $2.13 \mathrm{mmol} / \mathrm{L}, 7.08 \pm 3.60 \mathrm{mmol} / \mathrm{L}$, and $6.65 \pm 1.45 \mathrm{mmol} / \mathrm{L}$, respectively. Subjects carrying the T allele of SMARCA4 rs1122608 had higher glucose concentration $(p=0.019)$

Significant differences in serum LDL-C and HDL-C were seen among the 3 genotypes of rs 12232780 . The mean serum LDL-C was $1.84 \pm 0.69 \mathrm{mmol} / \mathrm{L}$ in the GG group, $1.67 \pm 0.58 \mathrm{mmol} / \mathrm{L}$ in the AA group ( $p=$ 0.012 ). Subjects carrying the $\mathrm{G}$ allele of $12232780 \mathrm{had}$ higher serum HDL concentrations $(p=0.047)$ : $1.13 \pm 0.25$ $\mathrm{mmol} / \mathrm{L}$ for the GG genotype, $1.14 \pm 0.26 \mathrm{mmol} / \mathrm{L}$ for the GA genotype, $0.95 \pm 0.14 \mathrm{mmol} / \mathrm{L}$ for the AA genotype.

We also found that PDW exist differences among the three genotypes of $\operatorname{rs} 1529729(p=0.043): 14.38 \pm 3.03$ for the TT genotype, $13.85 \pm 2.71$ for the TC genotype, $15.63 \pm 3.08$ for the $\mathrm{CC}$ genotype.

Used multiple comparison, seven tests were performed, a significance level was $P<0.0071(0.05 / 7)$ based on Bonferroni correction, significant differences in serum LDL-C and glucose concentration were seen among the three genotype of rs11879293 in SMARCA4 gene.

\section{DISCUSSION}

In a case-control study of incident CHD within the population based Chinese Han cohort, we evaluated the associations of the disease with four SNPs documented as associated with CHD. We identified that rs11879293 and rs 12232780 in the SMARCA4 gene was associated with a decreased risk of CHD. Meanwhile, we also found that significant differences in glucose concentrations with rs11879293 and rs1122608 different genotype. Serum LDL-C and HDL-C were seen among the 3 genotypes of rs 12232780 exist differences.

In our research, we found that SMARCA4 rs11879293 and rs12232780 seemed to have strong protective effects on the CAD. At present, there were no relevant reports on the rs11879293 and rs12232780 and coronary heart disease. SMARCA4 is located in chromosomal region of $19 \mathrm{p} 13.2$, and its protein is the important catalytic component of the SWI/SNF complexes. It is composed of multiple domains, a conserved C-terminal bromodomain, the less characterized $\mathrm{N}$-terminal region which have crucial effect on DNA binding, recruitment of SWI/SNF and the recognition of modified histone proteins [16]. SMRACA4 is located closely to the low-density lipoprotein receptor gene and disrupting chromatin structure regulates the transcription of various genes using the chemical energy of adenosine triphosphate hydrolysis [17]. Besides, we rarely know that the functional meaning of this marker of the $19 \mathrm{p} 13$ locus, but being associated with LDL-cholesterol levels, pathogenic SNP within the adjacent $L D L R$ could be in linkage disequilibrium with the 19p13 locus [18].

Pan H. et al. [19] found that rs11879293 associated with the risk of hepatocellular carcinoma is more pronounced in males, younger individuals $(<60 \mathrm{y})$, and nondrinkers. However, we did not found rs11879293 
associated with CHD by previous reports. Amankwah et al. [20] provide suggestive evidence that rs 12232780 in SMARCA4 variant in the chromatin remodeling machinery may be associated with an increased risk for oligodendroglioma. We all know that coronary heart disease risk factor consists of LDL-C elevated and HDL-C decreased. We also found that LDL-C exist difference among the 3 genotypes in rs11879293 and rs12232780. The level of LDL-C in mutation genotype was lower than the wild type, and therefore, we hypothesized that these rs11879293 and rs12232780 might affect the occurrence of coronary heart disease by affecting the level of LDL-C.

In 2009, Knouff et al. [21] discovered that rs1122608 was associated with myocardial infarction. This site is a high risk factor of myocardial infarction in the Russian population, but the correlation with myocardial infarction in Han population is not obvious [22]. This loci rs 1122608 was associated with LDL-C and coronary heart disease in Caucasian population [18]. In the research literature in Iran also suggested that rs 1122608 (GT or TT) associated with the severity of coronary heart disease [13]. While, in our research, no significant result exist between rs 1122608 polymorphism and Coronary heart disease. In future studies, we will consider that the SMARCA4 gene may function differently in varying disease mechanisms.

There were several limitations to our study. First, all the samples were from the Chinese Han population living in Xi'an city or its surrounding area and from the same hospital. There were a substantial number of confounding factors that may have caused type I errors (false-positive results) in our association study. Second, our samples included 456 cases and 685 healthy controls and we performed a power analysis that showed that the power of four SNPs was $<0.75$. Our samples included 456 cases and 685 healthy controls and we performed a power analysis that showed that the power of four SNPs was $<$ 0.75 . It is generally believed that the statistical validity of a study should be more than $75 \%$. If the sample size is too small will reduce the degree of confidence, that is, the effectiveness of the reduction, if not up to the standard results are not credible. To obtain a statistical power of $80 \%, 3657$ subjects with CHD and 5485 controls are required for rs11879293, and 2402 subjects with CHD and 3604 controls for rs 12232780 . At present, so many samples (3657 subjects with CHD and 5485 controls) are difficult to collect, and it is not possible to collect so many samples in the short term. In our research, it exist an issue of the small study population, and the low statistical power. However, we will continue to collect coronary heart disease and healthy population, increase the statistical power, and continue to study the effect of SMARCA4 gene on coronary heart disease

BMI is an important cardiovascular disease risk factor, and raised blood pressure, cholesterol, and glucose partly mediate its effects. Beyond that, smoking, hypertension, diabetes mellitus, dyslipidemia, chronic kidney disease, and obesity are also risk factors for coronary heart disease. So, these characteristics are important for case-control studies. But in our study, we mainly research the association between gene polymorphism and coronary heart disease, and analysis the influence of gene polymorphism on biochemical index. In the process of collecting samples, participants did not provide us with the information (e.g. smoking, hypertension, diabetes mellitus, dyslipidemia, chronic kidney disease, and obesity). But in the future, we will continue to collect samples, perfect the clinical information, comprehensive study of the impact of polymorphism on coronary heart disease.

In conclusion, our comprehensive analysis of SNPs in the SMARCA4 gene suggests that SMARCA4 genotypes and haplotypes are associated with CHD risk in Chinese Han population. In the future, we will continue to collect samples, perfect the clinical information, comprehensive study of the impact of polymorphism on coronary heart disease.

\section{MATERIALS AND METHODS}

\section{Ethics statement}

The protocol in this study conformed to the principles of the Declaration of Helsinki and was ratified by the Ethical Committee of the Second Affiliated Hospital of Xi'an Jiaotong University Health Science Center, China. Signed informed consent was obtained from each participant.

\section{Study participants}

In our study population, all analyses were restricted to Chinese Han population. We recruited a total of recently diagnosed confirmed 456 CHD patients from September 2012 to November 2014 into an ongoing molecular epidemiological study at the Second Affiliated Hospital of Xi'an Jiaotong University Health Science Center in Xi' an city, China. There were no age, sex, or disease stage restrictions for case recruitment. All patients were recently diagnosed confirmed to have CHD. Data collection for identification and validation of coronary events at baseline and throughout follow-up included the World Health Organization (WHO) chest pain questionnaire, Electrocardiogram(ECG)(coded using Minnesota Classification Code), self-reported doctor diagnosis of disease, record linkage to hospital discharge data and death certificates, and scrutiny of general practitioner records [23]. Questionnaire data were collected on history of diabetes blood pressure medication use, daily smoking, history of MI at baseline and family history of CHD [24]. In the current analyses, the classification of $\mathrm{CHD}$ was 
based on validated events and comprised of fatal or nonfatal myocardial infarction (MI), coronary artery bypass surgery, angioplasty, angina and/or unspecified ischaemic heart disease. We also collected the clinical data of CHD patient, including PLT, PCT, MPV, PDW, and Serum concentrations of ALT, AST, GGT, TP, GLU, TG, TC, HDL-C, LDL-C, APOA, APOB, Lp(a).

We also selected 685 healthy unrelated individuals between November 2012 and August 2014 from the medical examination center, the Second Affiliated Hospital of Xi' an Jiaotong University Health Science Center, who were recruited for scientific research into human complex diseases such as lung, stomach and liver chronic diseases. All the participants were restricted to Chinese Han who lived in Xi'an city and its surrounding areas.

\section{SNP identification}

We genotyped five single nucleotide polymorphisms (SNPs) with minor allele frequency (MAF) $>5 \%$ in SMARCA4 genes in the HapMap Asian population [12]. 5 $\mathrm{ml}$ venous blood was collected in a tube containing EDTA, and genomic DNA was extracted fromleukocytes using the GoldMag nanoparticles method (GoldMag Ltd. Xi'an, China) according to the manufacturer's instructions. DNA concentration was measured by spectrometry (DU530 UV/ VIS spectrophotometer; Beckman Instruments, Fullerton, CA, USA). We designed the Multiplexed SNP Mass EXTEND assay using Sequenom MassARRAY Assay Design version 4.0 software [25].

\section{Statistical analysis}

All continuous data are presented as means \pm standard deviations (SDs). Student $t$ test were used to compare the distribution of continuous variables. Differences in continuous variables among the subjects with three genotypes of related genes were assessed using the ANOVA. Statistical analyses were performed using SPSS 19.0 statistical package (SPSS, Chicago, IL). The genotype frequencies of each SNP in the control subjects were checked using the Hardy-Weinberg equilibrium (HWE). Power analysis was carried out using the online calculator at http://sampsize.sourceforge.net/iface/ s3.html. Data analysis was performed using SPSS version 16.0 statistical package (SPSS, Chicago, IL, USA) and Microsoft Excel. The significance of the difference of alleles and genotype frequencies between the groups was tested using the chi-square method [26]. $P<0.05$ was considered to represent statistical significance. Differences in the distribution were analyzed using logistic regression. Odds ratios (ORs) and 95\% confidence intervals (CIs) were tested using unconditional logistic regression analysis with adjustment for age and gender [27]. We also performed Bonferroni correction to assess the effect of genetic polymorphisms on coronary heart disease. Finally, we used the SHEsis software platform (www.nhgg.org/ analysis/) for analyses of LD, haplotype construction, and genetic association at polymorphism loci [28].

\section{ACKNOWLEDGMENTS}

This work were supported by the National Natural Science Foundation of China (Grant Nos. 81273878) and Social development of scientific and technological projects of Shaanxi Province (Grant Nos. 2016SF-339). We are also grateful to the clinicians and other hospital staff who contributed to the blood sample and data collection for this study.

\section{CONFLICTS OF INTERESTS}

The authors declare that they have no competing interests.

\section{REFERENCES}

1. Zhang XH, Lu ZL and Liu L. Coronary heart disease in China. Heart. 2008; 94:1126-1131.

2. Lopez AD, Mathers CD, Ezzati M, Jamison DT and Murray CJ. Global and regional burden of disease and risk factors, 2001: systematic analysis of population health data. Lancet. 2006; 367:1747-1757.

3. Bhasin SK, Dwivedi S, Dehghani A and Sharma R. Conventional risk factors among newly diagnosed coronary heart disease patients in Delhi. World journal of cardiology. 2011; 3:201-206.

4. Schunkert H, Erdmann J and Samani NJ. Genetics of myocardial infarction: a progress report. European heart journal. 2010; 31:918-925.

5. Rinkuniene E, Petrulioniene Z, Laucevicius A, Ringailaite E and Laucyte A. [Prevalence of conventional risk factors in patients with coronary heart disease]. Medicina. 2009; 45:140-146.

6. Schunkert H, Konig IR, Kathiresan S, Reilly MP, Assimes TL, Holm H, Preuss M, Stewart AF, Barbalic M, Gieger C, Absher D, Aherrahrou Z, Allayee H, et al. Large-scale association analysis identifies 13 new susceptibility loci for coronary artery disease. Nature genetics. 2011; 43:333-338.

7. Coronary Artery Disease C, Samani NJ, Deloukas P, Erdmann J, Hengstenberg C, Kuulasmaa K, McGinnis R, Schunkert H, Soranzo N, Thompson J, Tiret L and Ziegler A. Large scale association analysis of novel genetic loci for coronary artery disease. Arteriosclerosis, thrombosis, and vascular biology. 2009; 29:774-780.

8. McPherson R, Pertsemlidis A, Kavaslar N, Stewart A, Roberts R, Cox DR, Hinds DA, Pennacchio LA, TybjaergHansen A, Folsom AR, Boerwinkle E, Hobbs HH and Cohen JC. A common allele on chromosome 9 associated 
with coronary heart disease. Science. 2007; 316:1488-1491.

9. Coronary Artery Disease Genetics C. A genome-wide association study in Europeans and South Asians identifies five new loci for coronary artery disease. Nature genetics. 2011; 43:339-344.

10. Moes-Sosnowska J, Szafron L, Nowakowska D, DansonkaMieszkowska A, Budzilowska A, Konopka B, PlisieckaHalasa J, Podgorska A, Rzepecka IK and Kupryjanczyk J. Germline SMARCA4 mutations in patients with ovarian small cell carcinoma of hypercalcemic type. Orphanet journal of rare diseases. 2015; 10:32.

11. Wilson BG, Helming KC, Wang X, Kim Y, Vazquez F, Jagani Z, Hahn WC and Roberts CW. Residual complexes containing SMARCA2 (BRM) underlie the oncogenic drive of SMARCA4 (BRG1) mutation. Molecular and cellular biology. 2014; 34:1136-1144.

12. Fujimaki T, Oguri M, Horibe H, Kato K, Matsuoka R, Abe S, Tokoro F, Arai M, Noda T, Watanabe S and Yamada Y. Association of a transcription factor 21 gene polymorphism with hypertension. Biomedical reports. 2015; 3:118-122.

13. Jamaldini SH, Babanejad M, Mozaffari R, Nikzat N, Jalalvand K, Badiei A, Sanati H, Shakerian F, Afshari M, Kahrizi K and Najmabadi H. Association of polymorphisms at LDLR locus with coronary artery disease independently from lipid profile. Acta Med Iran. 2014; 52:352-359.

14. Liu Y, Zhou D, Zhang Z, Song Y, Zhang D, Zhao T, Chen Z, Sun Y, Zhang D, Yang Y, Xing Q, Zhao X, Xu H and He L. Effects of genetic variants on lipid parameters and dyslipidemia in a Chinese population. Journal of lipid research. 2011; 52:354-360.

15. Zhong R, Liu L, Tian Y, Wang Y, Tian J, Zhu BB, Chen W, Qian JM, Zou L, Xiao M, Shen N, Yang H, Lou J, et al. Genetic variant in SWI/SNF complexes influences hepatocellular carcinoma risk: a new clue for the contribution of chromatin remodeling in carcinogenesis. Scientific reports. 2014; 4:4147.

16. Singh M, D'Silva L and Holak TA. DNA-binding properties of the recombinant high-mobility-group-like AT-hookcontaining region from human BRG1 protein. Biological chemistry. 2006; 387:1469-1478.

17. Mulholland N, Xu Y, Sugiyama H and Zhao K. SWI/SNFmediated chromatin remodeling induces Z-DNA formation on a nucleosome. Cell \& bioscience. 2012; 2:3.

18. Kathiresan S, Willer CJ, Peloso GM, Demissie S, Musunuru K, Schadt EE, Kaplan L, Bennett D, Li Y, Tanaka T, Voight $\mathrm{BF}$, Bonnycastle LL, Jackson AU, et al. Common variants at 30 loci contribute to polygenic dyslipidemia. Nature genetics. 2009; 41:56-65.
19. Pan H, Niu DD, Feng H, Ng LF, Ren EC and Chen WN. Cellular transcription modulator SMARCE1 binds to HBV core promoter containing naturally occurring deletions and represses viral replication. Biochimica et biophysica acta. 2007; 1772:1075-1084.

20. Amankwah EK, Thompson RC, Nabors LB, Olson JJ, Browning JE, Madden $\mathrm{MH}$ and Egan KM. SWI/SNF gene variants and glioma risk and outcome. Cancer epidemiology. 2013; 37:162-165.

21. Myocardial Infarction Genetics C, Kathiresan S, Voight BF, Purcell S, Musunuru K, Ardissino D, Mannucci PM, Anand S, Engert JC, Samani NJ, Schunkert H, Erdmann J, Reilly MP, et al. Genome-wide association of early-onset myocardial infarction with single nucleotide polymorphisms and copy number variants. Nature genetics. 2009; 41:334341.

22. Wang Y, Wang L, Liu X, Zhang Y, Yu L, Zhang F, Liu L, Cai J, Yang $X$ and Wang $X$. Genetic variants associated with myocardial infarction and the risk factors in Chinese population. PloS one. 2014; 9:e86332.

23. Price JF, Lee AJ, Rumley A, Lowe GD and Fowkes FG. Lipoprotein (a) and development of intermittent claudication and major cardiovascular events in men and women: the Edinburgh Artery Study. Atherosclerosis. 2001; 157:241-249.

24. Nascetti S, Linarello S, Scurti M, Grandi E, Gaddoni M, Noera $G$ and Gaddi A. Assessment of the health status in the Massa Lombarda cohort: a preliminary description of the program evaluating cardio-cerebro-vascular disease risk factors and quality of life in an elderly population. Archives of gerontology and geriatrics Supplement. 2004; :309-314.

25. Trembizki E, Smith H, Lahra MM, Chen M, Donovan B, Fairley CK, Guy R, Kaldor J, Regan D, Ward J, Nissen MD, Sloots TP and Whiley DM. High-throughput informative single nucleotide polymorphism-based typing of Neisseria gonorrhoeae using the Sequenom MassARRAY iPLEX platform. The Journal of antimicrobial chemotherapy. 2014; 69:1526-1532.

26. Adamec C. [Example of the Use of the Nonparametric Test. Test X2 for Comparison of 2 Independent Examples]. Ceskoslovenske zdravotnictvi. 1964; 12:613-619.

27. Bland JM and Altman DG. Statistics notes. The odds ratio. Bmj. 2000; 320:1468.

28. Yong Y and Lin H. SHEsis, a powerful software platform for analyses of linkage disequilibrium, haplotype construction, and genetic association at polymorphism loci. Cell research. 2005; 15:97-98. 